\title{
Pneumatic Pressure Control: An Open-Source Droplet Microfluidic System
}

\author{
Run Ze Gao, Marie Hébert, and Carolyn L. Ren \\ Mechanical and Mechatronics Engineering \\ University of Waterloo \\ Waterloo, Canada \\ c3ren@uwaterloo.ca
}

\begin{abstract}
Open-source droplet microfluidic systems are envisioned to decrease the barriers to entry for exploration in new microfluidic research areas and increase adoptions in existing areas such as micro total analysis systems. At the heart of droplet microfluidics systems is a pneumatic pressure control system, which produces pulseless pressure-driven flow to manipulate nanoliter-sized droplet movement in microfluidic environments. Currently, this device remains a barrier regarding cost and performance. Commercially available off-the-shelf and customized systems offer high performance at a premium price and are proprietary which cannot be upgraded or modified. In-house built systems often do not exist due to long development cycles from the ground up. Hence, other than dedicated droplet microfluidics groups, compromises regarding the cost to performance ratio and development flexibility are taken by most.
\end{abstract}

This project presents a pneumatic pressure control system based on open-source hardware and software at a fraction of the cost and similar performance compared to premium commercial systems. All components are widely available through online suppliers. The code, design, and bill of material are made available for everyone on GitHub. The open-source nature of this project provides a gateway for many on a tight budget to tryout droplet microfluidics with the pulseless flow. This project is a small step in creating an open ecosystem for droplet microfluidics. Nonetheless, it enables an alternative to the rigid and non-standardized methodology that exists in the overarching microfluidics and lab on chip community today.

Keywords - Microfluidics, pnuematic control, open-source

\section{INTRODUCTION}

Fluigent [1], Elveflow [2] and Dolomite [3] produces premium pneumatic controlled actuators commercially. These systems have high performance but lack flexibility due to proprietary and closed-source nature of commercial products. The cost is also much higher than the open-source pneumatic pressure control system introduced here. This prevents the adoption of precision microfluidic control which is one the key area that must be developed for broad adoption of microfluidic and lab-on-chip technology.

\section{METHODOLOGY}

The making of the open-source pneumatic control system for microfluidics involves two critical factors, component selection and procedure for assembly and testing as it must be reproducible by others. Fig. 1 shows the open-source system development steps.

Component selection is crucial due to the very nature of open-source projects. All parts are selected to be easily obtainable and widely available via reputable online sellers. The procedure for assembly and performance testing are streamlined.

\section{A. Materials}

The components are purchased from online sellers, which are DigiKey, McMaster Carr, and Amazon. The pressure actuator is purchased from ControlAir. All components can be acquired worldwide.

\section{B. Procedure}

The building procedure will be outlined in the standard operating procedure available on GitHub. The complete bill of material, software code, printed circuit board/soldered breadboard design, housing design and performance testing procedure will be available on GitHub as well. All resources available on GitHub for this pneumatic pressure control

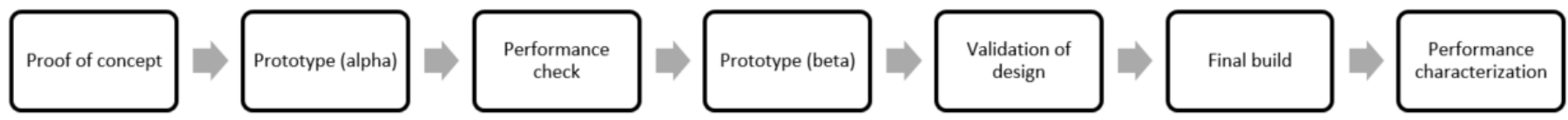

Figure 1. Open-source pneumatic pressure control system development process 


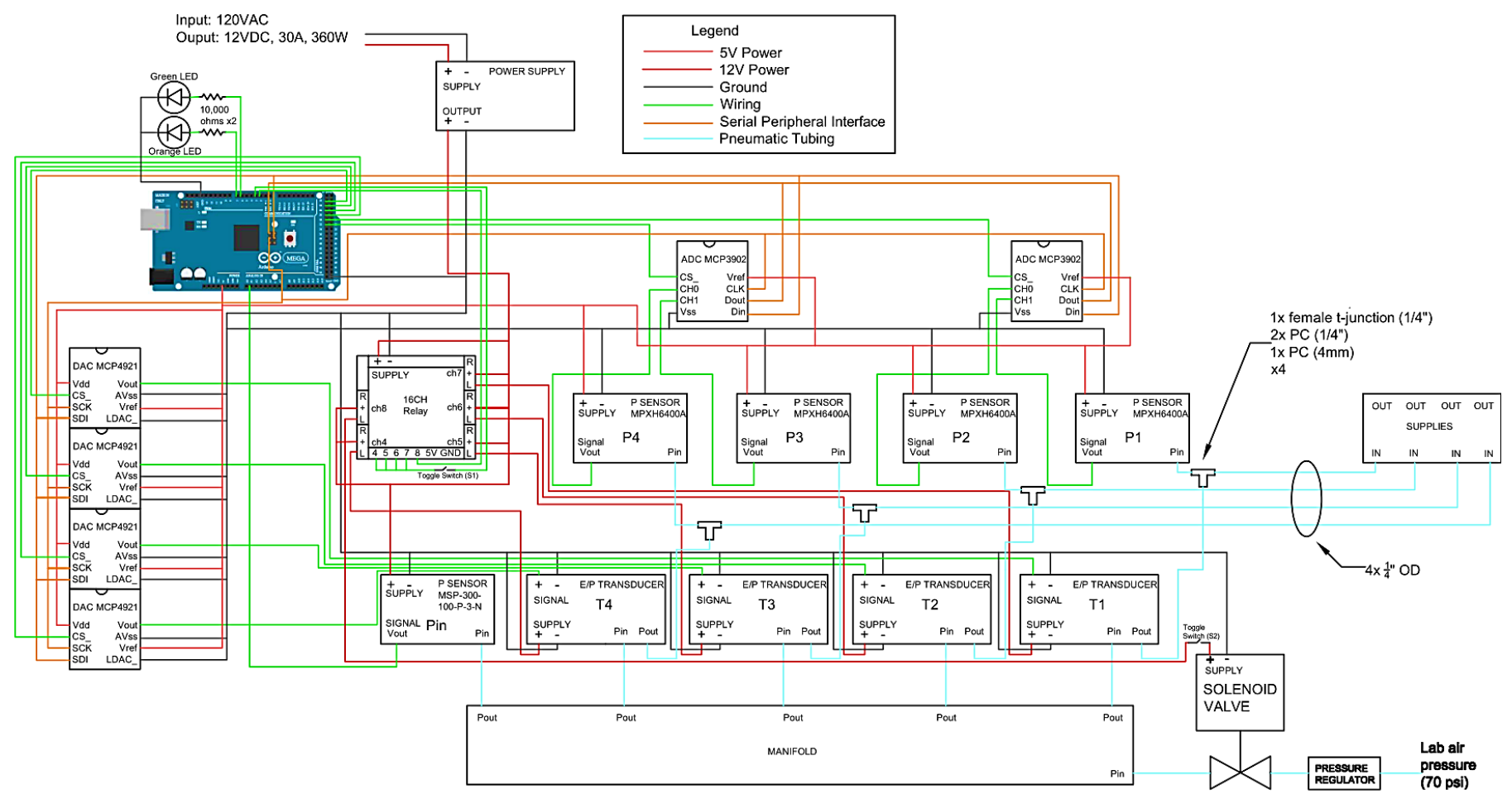

Figure 2. Open-source pneumatic pressure control system schematic

system will be open to everyone with General Public License.

The building procedure consists of populating the soldered breadboard or printed circuit board with electronic components, and system integration.

The performance and criteria validation procedure includes a signal to noise ratio testing, response testing, stability testing, and real-world performance testing.

\section{DESIGN}

Due to the open-source nature of the project, the performance to cost ratio is crucial. Ideally, the performance should be on par with the premium commercial systems while being much cheaper than commercial units. Table 1 and Table 2 shows the design constraints and criteria. Fig. 1 shows the design schematic.

Table 1. Pneumatic pressure control system design constraints

\begin{tabular}{|c|c|c|}
\hline $\begin{array}{c}\text { Design } \\
\text { Constraint }\end{array}$ & $\begin{array}{c}\text { Constraint } \\
\text { Value/Range }\end{array}$ & Description/Reasoning \\
\hline $\begin{array}{c}\text { Pressure } \\
\text { range }\end{array}$ & $\begin{array}{c}0 \text { to } \\
2000 \mathrm{mbar}\end{array}$ & $\begin{array}{c}\text { Most microfluidics operates within this } \\
\text { range. }\end{array}$ \\
\hline Resolution & $\leq 0.5 \mathrm{mbar}$ & $\begin{array}{c}\text { For most application's sensitivity } \\
\text { requirement, this resolution is sufficient. }\end{array}$ \\
\hline Accuracy & $\pm 5 \mathrm{mbar}$ & $\begin{array}{c}\text { Physical flow is not affected by accuracy } \\
\text { smaller than this (hypothesis). }\end{array}$ \\
\hline Precision & $\pm 5 \mathrm{mbar}$ & $\begin{array}{c}\text { Physical flow is not affected by precision } \\
\text { smaller than this (hypothesis). }\end{array}$ \\
\hline $\begin{array}{c}\text { Response } \\
\text { time }\end{array}$ & $\leq 1$ second & $\begin{array}{c}\text { Passive microfluidics does not require } \\
\text { stringent response time as it operates } \\
\text { mostly based on steady state pressure per }\end{array}$ \\
\hline
\end{tabular}

\begin{tabular}{|c|c|c|}
\hline $\begin{array}{c}\text { Settling } \\
\text { time }\end{array}$ & $\leq 1$ second & $\begin{array}{c}\text { channel. Active-control systems are the } \\
\text { same as it operates based on closed-loop } \\
\text { feedback control of droplet position. } \\
\text { Passive microfluidics does not require } \\
\text { stringent settling time for the same reason } \\
\text { as response time. Active-control systems } \\
\text { operate based on droplet location rather } \\
\text { than droplet velocity/acceleration, thus } \\
\text { settling time is determined to be sufficient } \\
\text { at this constraint. }\end{array}$ \\
\hline
\end{tabular}

Table 2. Pneumatic pressure control system design criteria

\begin{tabular}{|c|c|}
\hline $\begin{array}{c}\text { Design } \\
\text { Criteria }\end{array}$ & Description/Reasoning \\
\hline $\begin{array}{c}\text { Safe to } \\
\text { operate }\end{array}$ & $\begin{array}{c}\text { The system must have redundancy built-in to ensure } \\
\text { electrical safety and pneumatic pressure safety for users. }\end{array}$ \\
\hline Pulseless & $\begin{array}{c}\text { The control system must produce pulseless flow during } \\
\text { real-world operations in any microfluidics systems with } \\
\text { any fluid, }\end{array}$ \\
\hline $\begin{array}{c}\text { Modular } \\
\text { hardware } \\
\text { design }\end{array}$ & $\begin{array}{c}\text { Allow the user to build the system, change components, } \\
\text { and upgrade hardware with ease }\end{array}$ \\
\hline $\begin{array}{c}\text { Open source } \\
\text { software }\end{array}$ & $\begin{array}{c}\text { The open source software allows the user to continuously } \\
\text { improve the software, and use any suitable control } \\
\text { algorithm, and integrate with their system seamlessly. }\end{array}$ \\
\hline $\begin{array}{c}\text { Ease of } \\
\text { maintenance }\end{array}$ & $\begin{array}{c}\text { The user has the flexibility to carry out maintenance of } \\
\text { any hardware by themselves with no patent or proprietary } \\
\text { restrictions. }\end{array}$ \\
\hline
\end{tabular}

\section{A. Control System and Data Acquisition}

The control system consists of an Arduino Mega, which is open-source electronic incorporating a microcontroller ATmega 2560, and four MCP 4921 which are single channel 12-bit digital to analog converters (DAC). The DAC converts 
digital control signals to analog signals for pneumatic pressure actuation through ControlAir T900.

The data acquisition system consists of two MCP 3202, which are two channel analog to digital converter (ADC) that converts analog signals from the external pressure sensor, MPX4250D, to digital signals feed into Arduino microcontroller used in feedback in the active-controlled microfluidic system, and user interface display in both passive and active-controlled microfluidic systems. Fig. 2 shows the full schematic of the four channel open-source pneumatic pressure control system.

\section{B. Pneumatic System}

The pneumatic system consists four pressure actuators, ControlAir T900, which has a range of 0 to 2000mbar, with \pm 2 mbar accuracy. Four pressure sensors, MPX4250D, are used to measure the pressure provided by the actuators, one sensor for each actuator. MPX4250D has a range of 0 to 2500 mbar, with an overall minimum accuracy of \pm 34.5 mbar. Various tubing is used to deliver pneumatic pressure to fluid reservoirs, which will be included in the bill of material on GitHub.

\section{Software and User Interface}

The software is written in $\mathrm{C}++$. Arduino IDE is used for the control and operation algorithm of the open-source pneumatic pressure control system. This includes the system start-up sequence, system calibration sequence, a signal converting algorithm, serial peripheral interfacing, pressure command code for the pressure actuators, and pressure read code for the pressure sensors.

The graphical user interface (GUI) and computer-tomicrocontroller communication are coded using MS Visual Studios based on QT framework. The design of the GUI is based on end-user suggestion as well as emulating premium commercial unit's GUI. GUI is designed to be minimalistic to streamline the user experience. However, due to the opensource nature of the software, the GUI can be modified with additional or fewer functions, and the GUI can be integrated with other Windows-based operating system GUI. Fig.3 shows the graphical user interface.

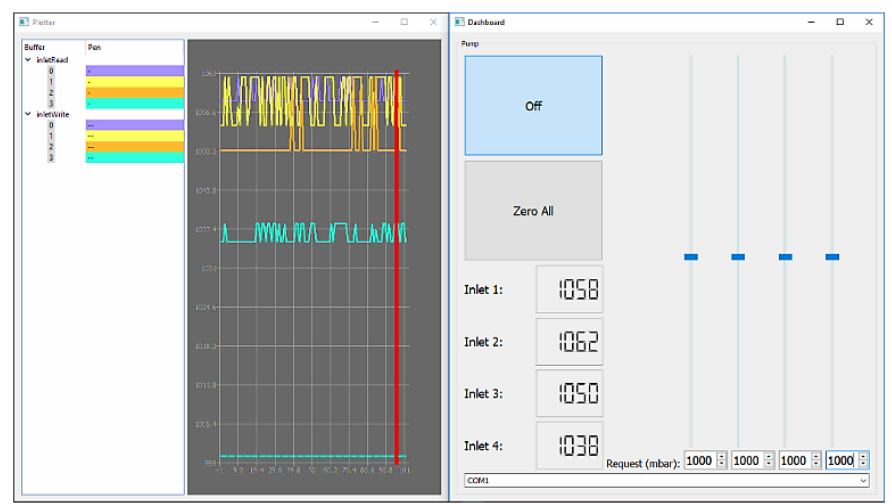

Figure 3. Open-source pneumatic pressure control system graphic user interface

\section{TESTING AND VALIDATION}

The signal testing is done using digital phosphor oscilloscope. The pressure performance testing and benchmarking is done using the prototype setup. Two different types of pressure sensors, MPX4250DP and MPXV5004 are used to conduct performance testing. A flow sensor, Sensirion SLG1430-480 is used to conduct real-world performance testing and benchmarking.

\section{A. Signal Noise Ratio}

Noise is minimized by using eigth-order Butterworth, low pass filters, MAX7480, which provides ADC anti-aliasing and post-DAC filtering. Also, noise is further reduced by using precision $5 \mathrm{~V}$ voltage reference, LM4040, which has an accuracy of $5 \mathrm{mV}$. The result of which is that the pressure sensors and pressure actuators are operating within their manufacture's specifications.

\section{B. Response Time}

Fig. 4 shows the step response time of all four transducers increasing from $1000 \mathrm{mbar}$ to $2000 \mathrm{mbar}$. The response time for each of the four transducers over this range is less than one second, which satisfies the response time and settling time constraints.

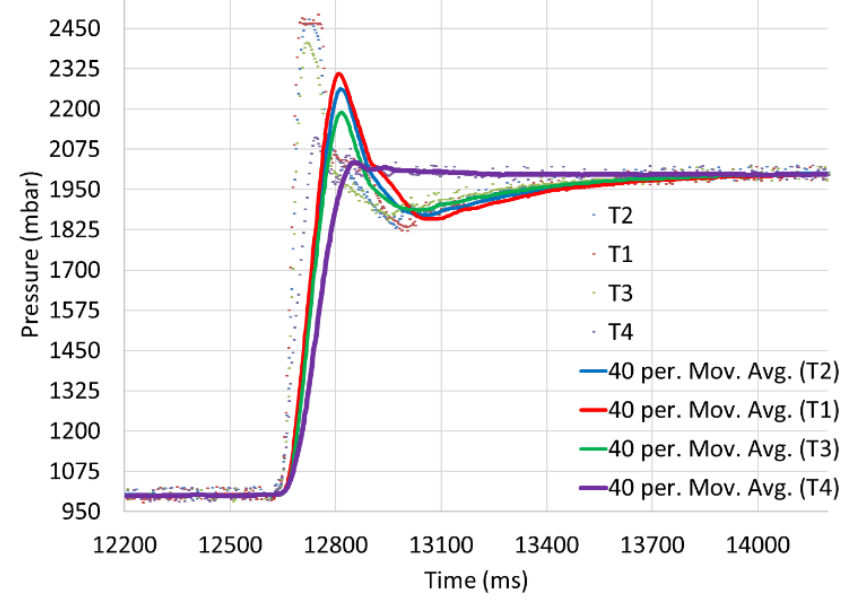

Figure 4. Response time and settling time of all four channels of the open-source pneumatic pressure control system

\section{Pressure Stability}

Fig. 5 shows the pressure stability comparison between one of pneumatic pressure control system's channels and one of Fluigent MFCS EZ's channels. The data is taken at $20 \mathrm{mbar}$. The two systems have similar performance regarding pressure stability. The accuracy and precision design constraints for pneumatic pressure control system are achieved. 


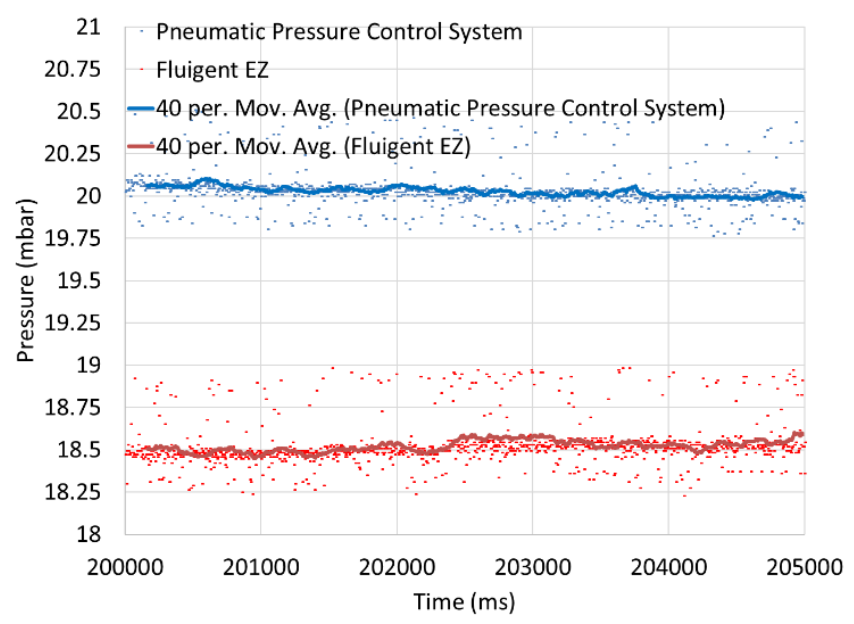

Figure 5. Pressure stability comparison between open-source pneumatic pressure control system and Fluigent MFCS EZ

\section{Flow Rate Stability}

Fig. 6 shows the flow rate stability comparison between one of pneumatic pressure control system's channels and one of Fluigent MFCS EZ's channels. The flow rate data are taken at $300 \mathrm{mbar}$. Both systems display similar flow rate stability.

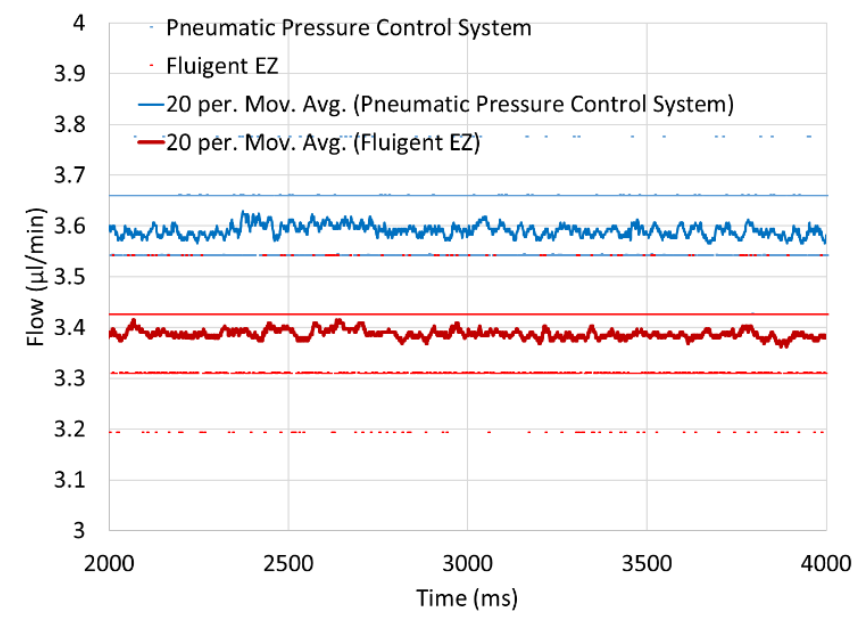

Figure 6. Flow Rate stability comparison between open-source pneumatic pressure control system and Fluigent MFCS EZ

\section{DISCUSSION}

Safety features are built into the design. Soft stops are incorporated into the code which stops the communication when the pressure sensor reads sudden unexpected pressure spikes or drops or higher than operating limit pressure levels. Hard stops include physical toggle switches that shut the supply power to the pneumatic solenoid and pressure actuators.

The testing and validations results show that the opensource pneumatic pressure control system can take full advantage of pressure actuator performance without sacrifice or compromise due to an analog signal to noise ratio. In other words, the precision and accuracy are limited by the pressure actuator. Also, precision is determined to be more important than accuracy as pulseless criteria is mostly concerned with precision. Overall, all of the design constraints and criteria are met.

The pressure stability test and flow rate stability test show that the open-source pneumatic pressure control system can achieve the same performance as Fluigent MFCS EZ. The difference between the two systems lies in the consistency in performance between channels/pressure actuators.

\section{CONCLUSION}

Safety features regarding pneumatic pressure and electricity have been incorporated into the design and build of the opensource pneumatic pressure control system. The testing and validation results show that the open-source pneumatic pressure control system can take full advantage of pressure actuator performance. Overall, all of the design constraints and criteria are met. The benchmarking shows that open-source pneumatic pressure control system has similar performance compared to Fluigent MFCS EZ while costing much less than a commercial system.

\section{ACKNOWLEDGMENT}

The authors would like to thank Natural Science and Engineering Research Council, Canada, and Ontario Center of Excellence for the grants awarded to Professor Carolyn Ren.

\section{REFERENCES}

[1] C.-A. Kieffer, S. Ritty, T. Boudot, N. Petit, J. Weber and A. Le Nel, "A High Precision Fluid Handling System Based on Pressure Actuation," in 3rd European Conference on Microfluidics, Heidelberg, 2012.

[2] Elveflow, "OB1 MK3 - Microfluidic Flow Control System," Elveflow, 2018. [Online]. Available: https://www.elveflow.com/microfluidic-flowcontrol-products/flow-control-system/pressure-controller/. [Accessed 13 03 2018].

[3] Dolomite, "Mitos P-Pump Basic," Dolomite, 2018. [Online]. Available: https://www.dolomite-microfluidics.com/product/mitos-p-pump-basic/. [Accessed 1303 2018]. 